\title{
A New Method to Construct Lower Bounds for Van der Waerden Numbers
}

\author{
P.R. Herwig, M.J.H. Heule, \\ P.M. van Lambalgen, H. van Maaren \\ Department of Electrical Engineering, \\ Mathematics and Computer Science \\ Delft University of Technology, The Netherlands \\ P.R.Herwig@ewi.tudelft.nl, marijn@heule.nl, \\ P.M.vanLambalgen@gmail.com, H.vanMaaren@ewi.tudelft.nl
}

Submitted: Nov 1, 2005; Accepted: Dec 18, 2006; Published: Jan 3, 2007

Mathematics Subject Classification: 05D10

\begin{abstract}
We present the Cyclic Zipper Method, a procedure to construct lower bounds for Van der Waerden numbers. Using this method we improved seven lower bounds. For natural numbers $r, k$ and $n$ a Van der Waerden certificate $W(r, k, n)$ is a partition of $\{1, \ldots, n\}$ into $r$ subsets, such that none of them contains an arithmetic progression of length $k$ (or larger). Van der Waerden showed that given $r$ and $k$, a smallest $n$ exists - the Van der Waerden number $W(r, k)$ - for which no certificate $W(r, k, n)$ exists. In this paper we investigate Van der Waerden certificates which have certain symmetrical and repetitive properties. Surprisingly, it shows that many Van der Waerden certificates, which must avoid repetitions in terms of arithmetic progressions, reveal strong regularities with respect to several other criteria. The Cyclic Zipper Method exploits these regularities. To illustrate these regularities, two techniques are introduced to visualize certificates.
\end{abstract}

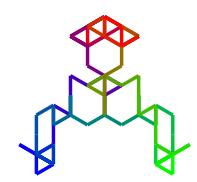

${ }^{*}$ Supported by the Dutch Organization for Scientific Research (NWO) under grant 617.023.306 


\section{Introduction}

In 1927 the Dutch mathematician Van der Waerden proved [18] a (generalization of) a conjecture of Schur ${ }^{1}$ : For given numbers $r$ and $k$, there exists a smallest number $n$ - the Van der Waerden number $W(r, k)$ - such that each partition of the set $\{1,2, \ldots, n\}$ into $r$ subsets contains at least one subset with an arithmetic progression of at least length $k$. An arithmetic progression of length $k$ is a sequence of $k$ numbers, such that the differences between consecutive numbers is a constant $d$. For example, the set $\{a, a+d, a+2 d, \ldots, a+$ $(k-1) d\}$ is an arithmetic progression of length $k$. At present only five ${ }^{2}$ of the smaller Van der Waerden numbers are known $[1,3,16]$. These numbers were obtained by using computational power.

Considerable effort has been invested into establishing good estimates for the Van der Waerden numbers. The original proof of Van der Waerden bounded the numbers above by an Ackermann function in $k$. Such a function grows faster than any primitive recursive function. Only since the proof of Shelah [14] in 1986, the Van der Waerden numbers are known to be bound above by a primitive recursive function. Gowers [8] has tightened these upper bounds even more by providing an alternative proof of the Szemerédi theorem [17] on arithmetic progressions.

Still, there is a significant gap between the upper and lower bounds on the Van der Waerden numbers. The best function binding the Van der Waerden numbers below is exponential in $k$. Several general results for lower bounds are known. The first proofs, by Erdős and Rado [5], were non-constructive and applied probabilistic methods. Berlekamp [2] was the first to publish a construction for lower bounds based on purely algebraic arguments. Rabung [13] improved some of these bounds, but he could not provide a generalizable construction. This latter article has gone largely unnoticed: Most tables in articles, books and on the Internet ignore it. More recently, satisfiability (SAT) solving techniques have been used to improve lower bounds. See for example Dransfield, Liu, Marek and Truszczynski [4]. A new lower bound for $W(5,3)$ was discovered using their method. Also Kouril and Franco [11] used SAT to establish an improved lower bound for $W(2,6)$. Using SAT solvers seems a promising method for this purpose.

Since the authors stem from the SAT solving community, and since searching for Van der Waerden certificates is easily formulated as a SAT problem, our first motivation to this study was to discover whether the tremendous progress in SAT solving techniques in other areas, would extend to the search for Van der Waerden numbers. Especially new CNF loading techniques (which could enhance solving performance, compared to [7], [11]) seemed promising at first sight. The latter because admissible Van Der Waerden certificates exhibit regularities of a certain kind, which could be forced to extrapolate to larger instances, thus creating the possibly of finding larger certificates, of course without any implication on upper bound features. Improved lower bounds to Schur numbers were also established by forcing patterns [6].

\footnotetext{
${ }^{1}$ In his original paper Van der Waerden refers to Baudet and Artin as origin.

${ }^{2}$ Recently Kouril claims to have found a sixth Van der Waerden number: $W(2,6)=1132$.
} 
And in fact, it seemed possible to find larger certificates by intuitive constructions, which however immediately revealed certificates and hence the SAT search aspect turned out to be of no extra use: Another example of the victory of human creativity over automated search - although the latter seemed successful at least in recently establishing $W(2,6)=1132$ after 253 days of computational time.

We present the constructions as they were carried out. Verification of validity however is not possible by a traditional Mathematical proof. This verification is computer-aided. We will provide the reader the rough data of the certificates, to make verification reproducible.

This paper focuses exclusively on lower bounds. Its main topic, the Cyclic Zipper Method, originates from the SAT approach: We combine our observations regarding certain symmetric and repetitive properties of Van der Waerden certificates produced by these SAT solving techniques with some existing techniques. By using the Cyclic Zipper Method, seven lower bounds were improved substantially.

The next section of this paper provides the necessary theorems and definitions, along with the current best known lower bounds. In section 3, some regularities in certificates are discussed. In section 4, we present three methods to obtain so called cyclic certificates. In section 5, the results of our method are presented, including all improved lower bounds. In section 6, we conclude with an evaluation of the results.

\section{Preliminaries}

\subsection{Definition of Van der Waerden numbers}

Van der Waerden numbers, first introduced by Van der Waerden [18], arise from the following theorem:

Theorem 2.1 (Van der Waerden) Given two positive integers $r$ and $k$, there exists a smallest number $W(r, k)$ with the following property:

For each partition $\left\{1,2, \ldots, n_{0}\right\}=C_{1} \cup C_{2} \cup \ldots \cup C_{r}$ (with $n_{0} \geq W(r, k)$ ) there is at least one $C_{i}$ which contains an arithmetic progression of length at least $k$.

An arithmetic progression of length $k$ is a progression of numbers $a, a+d, a+2 d, \ldots, a+$ $(k-1) d$ for some $d>0$.

Definition 2.1 A Van der Waerden certificate $W(r, k, n)$ is a partition of $\{1,2, \ldots, n\}$ into $r$ subsets, none of which contains an arithmetic progression of length $\geq k$.

The latter is equivalent to stating that $W(r, k)>n$. A certificate $W(r, k, n)$ therefore provides a lower bound $n$ for the Van der Waerden number $W(r, k)$. 


\subsection{Current bounds of Van der Waerden numbers}

Only five smaller Van der Waerden numbers are known at present. The known Van der Waerden numbers, as well as the best known lower bounds and their sources are summarized in table 1 .

Table 1: Known Van der Waerden numbers and previously best known lower bounds.

\begin{tabular}{|c|r|r|c|c|c|c|c|}
\hline$r \backslash k$ & \multicolumn{1}{|c|}{$\mathbf{3}$} & $\mathbf{4}$ & $\mathbf{5}$ & $\mathbf{6}$ & $\mathbf{7}$ & $\mathbf{8}$ & $\mathbf{9}$ \\
\hline \hline $\mathbf{2}$ & $9[3]$ & $35[3]$ & $178[16]$ & $>1131[11]$ & $>3703[13]$ & $>7484[13]$ & $>27113[13]$ \\
\hline $\mathbf{3}$ & $27[3]$ & $>292[13]$ & $>965[13]^{3}$ & $>8886[13]$ & $>43855[13]$ & $>238400[13]^{4}$ & \\
\hline $\mathbf{4}$ & $76[1]$ & $>1048[13]$ & $>10437[13]$ & $>90306[13]$ & $>387967[13]^{4}$ & & \\
\hline $\mathbf{5}$ & $>125[4]$ & $>2254[13]$ & $>24045[13]$ & $>246956[13]^{4}$ & & & \\
\hline $\mathbf{6}$ & $>207[13]$ & $>9778[13]$ & $>56693[13]^{4}$ & $>600486[13]^{4}$ & & & \\
\hline
\end{tabular}

\section{Regularities in certificates}

Many Van der Waerden certificates known turn out to exhibit some form of regularity. To illustrate these regularities, we first introduce two methods to visualize certificates. The latter part of this section describes the three most occurring patterns.

\subsection{Graphical representations}

As an introduction to our observations of certain symmetries and repetitions of Van der Waerden certificates, we consider the following question: Is it possible to partition set $A=\{1, \ldots, 17\}$ into three subsets $A=C_{1} \cup C_{2} \cup C_{3}$ in such a way that no subset contains an arithmetic progression of length four? Any certificate $W(3,4,17)$ guarantees that $W(3,4)>17$. A valid partition is for example:

$$
\begin{aligned}
& C_{1}=\{1,3,11,13,15,16\} \\
& C_{2}=\{2,4,5,8,17\} \\
& C_{3}=\{6,7,9,10,12,14\}
\end{aligned}
$$

It proves insightful to depict the problem graphically by creating a $r \times n$ grid, with the rows representing the different subsets $C_{i}$. A black filled square in the $j$-th column of row $i$ denotes number $j$ is contained in subset $C_{i}$. By definition, each number $\{1,2, \ldots, n\}$ is contained in exactly one subset $C_{i}$.

However, it is difficult to visualize larger certificates this way. It also does not reveal certain patterns in the certificates easily. A different type of visualization shows the emerging patterns more clearly. When $r$-partitioning is involved we use $r$ directions in the plane, where the angle between two consecutive directions is $\frac{360^{\circ}}{r}$. Starting from the

\footnotetext{
${ }^{3}$ Landman and Robertson [12] refer to an untraceable lower bound $W(3,5)>1209$.

${ }^{4}$ Unpublished lower bounds which could be established using the method presented in [13].
} 
beginning of the certificate a line segment is drawn in the direction associated with the subset containing number 1 . From the endpoint of that line segment a line segment with equal length is drawn in the direction associated with the subset containing number 2 . This process is repeated up to number $n$ of the certificate. The line segments are gradually colored from red to blue to green and back to red. This visualization is only applicable for $r>2$. Both representations of the example certificate $W(3,4,17)$ are shown in figure 1 .
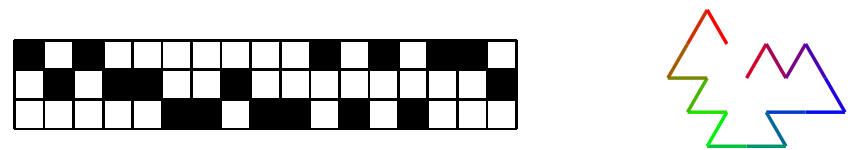

Figure 1: Graphical representations of a $W(3,4,17)$.

\subsection{Patterns}

When observing the largest known Van der Waerden certificates, it shows that they admit certain patterns. Patterns that occur often are:

\subsubsection{Symmetry}

Given a partition $\{1, \ldots, n\}=C_{1} \cup \cdots \cup C_{r}$, we refer to the reverse of subset $C_{i}$, denoted as $\bar{C}_{i}$ as:

$$
\bar{C}_{i}:=\left\{\begin{array}{l}
\left\{n+1-j \mid j \in C_{i}, \text { for } j=1, \ldots, n\right\} \quad \text { if } n \text { is even } \\
\left\{n+2-j \mid j \in C_{i}, \text { for } j=2, \ldots, n\right\} \text { if } n \text { is odd }
\end{array}\right.
$$

A certificate $W(r, k, n)$ is called point symmetric, denoted by $P W(r, k, n)$, if there exists a permutation $\pi$ of the subsets such that $\bar{C}_{i}=C_{r+1-i}$ if $n$ is even, and $\bar{C}_{i}=C_{r+1-i} \backslash\{1\}$ if $n$ is odd (for $i=1, \ldots, r$ ). For visualization purposes we assume permutation $\pi$ is applied for all $P W(r, k, n)$. Like certificates $W(r, k, n)$ : If there exists no certificate $P W(r, k, n)$, then there does not exist a certificate $P W(r, k, n+i)$ for $i>0$. Both graphical representations of a point symmetric certificate $P W(5,3,40)$ are shown in figure 2. Notice that the grid visualization is a point symmetric image, while the colored visualization has a reflection symmetry.
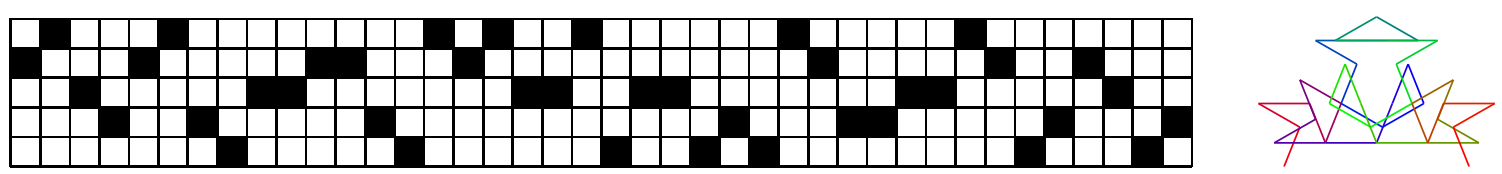

Figure 2: Graphical representations of a $P W(5,3,40)$.

A certificate $W(r, k, n)$ is called reflection symmetric, denoted by $R W(r, k, n)$, if $\bar{C}_{i}=C_{i}$ if $n$ is even, and $\bar{C}_{i}=C_{i} \backslash\{1\}$ if $n$ is odd (for $\left.i=1, \ldots, r\right)$. Like certificates $W(r, k, n)$ : If 
there exists no certificate $R W(r, k, n)$, then there does not exist a certificate $R W(r, k, n+i)$ for $i>0$ either. An example of a reflection symmetric certificate is the $R W(3,3,26)$ depicted below, which is the largest possible certificate: $W(3,3)=27$ - see figure 3 . Notice that - similar to the visualization of a point symmetric certificate - the visualization of a reflection symmetric certificate results in a reflection symmetric grid image and a point symmetric colored image.
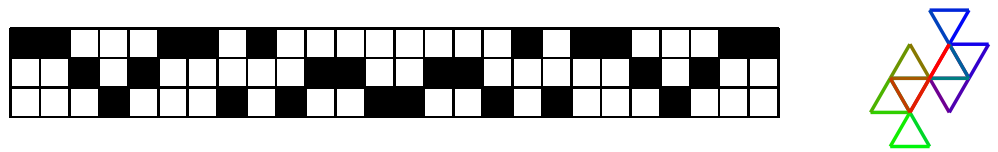

Figure 3: Graphical representations of a $R W(3,3,26)$.

\subsubsection{Repetition}

Apart from these symmetric properties, certificates can also be cyclic.

Definition 3.1 A cyclic certificate $c W(r, k, n)$ is a certificate which remains a certificate, for each $m$, under the transformation $j:=j+m(\bmod n)$ on the numbers $\{j=1, \ldots, n\}$ of the partition. The transformation involved is called a circular translation.

Cyclic certificates have the favorable property that they can be repeatedly appended to create larger certificates. A cyclic certificate $c W(r, k, n)$ can be repeated $(k-1)$ times to generate a certificate of length $n(k-1)$. For proof of this statement we refer to [15]. Due to this repetitive property, cyclic certificates will prove to be very valuable in the search for high Van der Waerden lower bounds. Following Rabung [13], one additional number can be added to the set $C_{r}$. A repetitive cyclic certificate is defined as:

Definition 3.2 A repetitive cyclic certificate $C W(r, k, n(k-1)+1)$ consists of $(k-1)$ appended cyclic certificates of length $n$ and one additional number.

Figure 4 (below) shows a visualization of a repetitive cyclic point symmetric certificate $C P W(2,4,34)$. It provides the largest possible lower bound for $W(2,4)$.

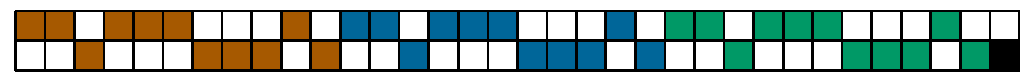

Figure 4: Graphical representation of a $C P W(2,4,3 \times 11+1)$.

\section{Constructing cyclic certificates}

Cyclic certificates, as defined in section 3.2.2, can be extended to certificates of larger size. Except for $W(2,3), W(3,3)$, and $W(5,3)$, all largest possible / known certificates are repetitive cyclic certificates. By focusing on obtaining only cyclic certificates, one could reduce the search space and possibly establish larger certificates. 


\subsection{Satisfiability solving}

The construction of a Van der Waerden certificate can easily be formulated as a satisfiability (SAT) problem. As mentioned in the introduction SAT solving techniques have recently been used to establish improved lower bounds [4, 11]. The SAT formulation of certificate $W(r, k, n)$ consists of $r \times n$ Boolean variables $x_{i, j}$. Each variable $x_{i, j}$ denotes the truth-value whether number $j$ belongs to subset $C_{i}$. The required clauses can be split in two types: (1) Clauses that force each number $j$ to be in exactly one subset $C_{i}$; and (2) clauses that forbid numbers in a subset to form an arithmetic progression of length $k$. For a detailed description of these constraints we refer to [4].

By using additional constraints - also known as streamlining [7] or tunnelling [11] patterns can be forced to reduce the search space. Point symmetry can be forced by adding binary equivalences $x_{i,\left\lceil\frac{n}{2}\right\rceil-j+1} \leftrightarrow x_{r-i+1,\left\lceil\frac{n}{2}\right\rceil+j}$ for all $i=1, \ldots, r$ and $j=1, \ldots,\left\lfloor\frac{n}{2}\right\rfloor$. Likewise, reflection symmetry can be forced by adding binary equivalences $x_{i,\left\lceil\frac{n}{2}\right\rceil-j+1} \leftrightarrow$ $x_{i,\left\lceil\frac{n}{2}\right\rceil+j}$ for all $i=1, \ldots, r$ and $j=1, \ldots,\left\lfloor\frac{n}{2}\right\rfloor$. Finally, cyclic certificates can be obtained by adding constraints of type (2). Forcing both a symmetry and repetition even further reduces the search space.

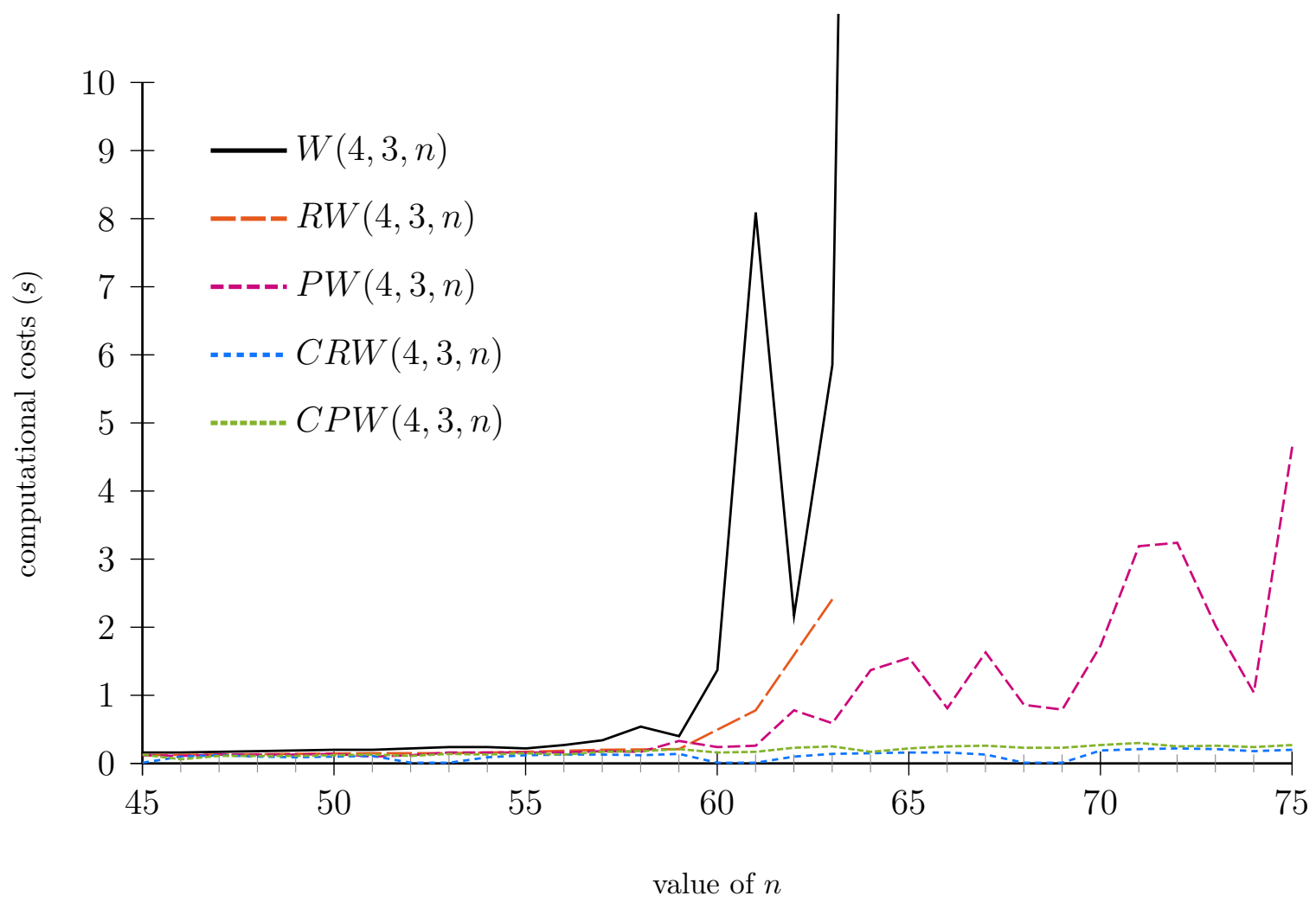

Figure 5: Costs to compute $W(4,3, n)$ by using some forced patterns. 
We studied the influence of adding forced patterns to reduce the computational costs to construct valid certificates. During the experiments, we used the SAT solver march_dl ${ }^{5}$ [10] to solve the generated formulas. Some of the results are shown in figure 5 . Recall that $W(4,3)=76$, so certificates $W(4,3, n)$ exist for $n \leq 75$. Notice that the computational costs to construct a certificate without a forced pattern requires much more time for larger $n$ : When $n$ gets closer to 75 , these costs increase up to thousands of seconds.

Several of the generated formulas with forced patterns appeared unsatisfiable, meaning no valid certificate exists of that kind. Notice that without requiring these regularities, unsatisfiability would mean an upper bound. Certificates $P W(4,3, n)$ exist for $n \leq 74$, while certificates $R W(4,3, n)$ exist only for $n \leq 62$. Most of the larger cyclic certificates were unsatisfiable. However, there exists a $C P W(4,3,75)$ which can be computed in 0.2 seconds. So, the largest possible certificate for $W(4,3)$ can be constructed while forcing patterns. This significantly reduces the computational cost to compute the ultimate lower bound. However, by adding constraints no upper bound can be computed for the original Van der Waerden problem.

\subsection{Power residue coloring}

In 1979, Rabung used power residues to construct Van der Waerden certificates. For the complete theorem and its proof we refer to [13]. We denote by $\rho_{p}$ the primitive root of unity of a prime $p$. Set $\{1, \ldots, p\}$ can be partitioned using this method by placing $j \in C_{i}$ such that

$$
C_{i}=\bigcup_{q=0}^{\frac{p-1}{r}-1} \rho_{p}^{i+q r}(\bmod p)+1 \quad(\text { for } i=1, \ldots, r)
$$

The potential certificate has to be validated. As an example, prime $37\left(\rho_{37}=2\right)$ is used to find a certificate $W(4,3,37)$ - see table 2 .

Table 2: Power residue coloring (partitioning) of 37 over 4 rows.

\begin{tabular}{|l|r|r|r|r|r|r|r|r|r|}
\cline { 2 - 10 } \multicolumn{1}{c|}{} & $q=0$ & $q=1$ & $q=2$ & $q=3$ & $q=4$ & $q=5$ & $q=6$ & $q=7$ & $q=8$ \\
\hline$C_{1}: \rho_{37^{1+4 q}}(\bmod 37)+1$ & 3 & 33 & 32 & 16 & 19 & 30 & 21 & 25 & 15 \\
\hline$C_{2}: \rho_{37^{2+4 q}}(\bmod 37)+1$ & 5 & 28 & 26 & 31 & 37 & 22 & 4 & 12 & 29 \\
\hline$C_{3}: \rho_{37^{3+4 q}}(\bmod 37)+1$ & 9 & 18 & 14 & 24 & 36 & 6 & 7 & 23 & 20 \\
\hline$C_{4}: \rho_{37^{4+4 q}}(\bmod 37)+1$ & 17 & 35 & 27 & 10 & 34 & 11 & 13 & 8 & 2 \\
\hline
\end{tabular}

Additionally, number 1 will be put in $C_{1}$. Graphical representations of this certificate are shown in figure 6 . Notice that the third and fourth row are interchanged to show the point symmetry. This is inherent to power residue coloring. Other certificates created by this method are also frequently point or reflection symmetric.

\footnotetext{
5 available from http://www.st.ewi.tudelft.nl/sat/
} 
Moreover, this certificate is cyclic and can be repeated $k-1$ times to produce a certificate of length 74. Adding one additional number results in a certificate of length 75 . This is the largest possible certificate $W(4,3, n): W(4,3)=76$. Except for distributing the numbers 1, 38 and 75 and permutations of the subsets, this is the only certificate $W(4,3,75)[4]$.
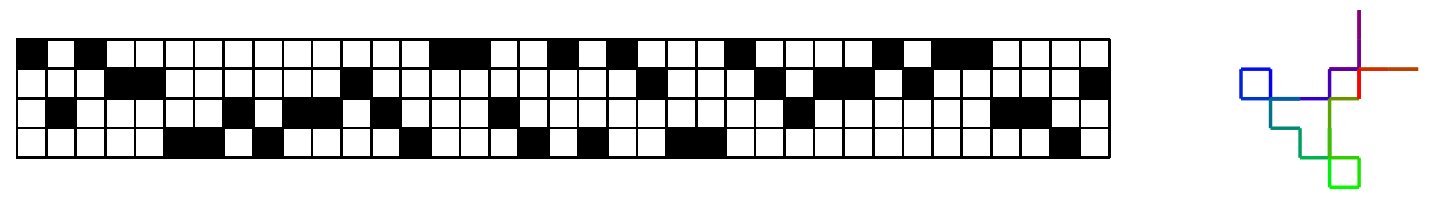

Figure 6: Graphical representations of a $P W(4,3,37)$ created with power residue coloring.

\subsection{Zipping}

Using the zipping technique, one can expand an existing cyclic certificate into a cyclic certificate of multiplied size. The basic concept is to zip two certificates into each other, creating a certificate of double size. As an example the a cyclic certificate $c P W(6,3,19)$ - see figure $7(a)$ - is zipped and the process is illustrated step by step. For traceability, another color is assigned to the different quadrants and the first number of the certificate.

First, the numbers are spread on all odd positions of a partition of double length: Figure $7(b)$. Second, another partition is created by turning the rows upside down: Figure $7(c)$. Third, this partition is shifted for the length of the original certificate to the left: Figure $7(d)$. Finally, in figure $7(e)$, the zipped certificate is shown as a result of merging figure $7(b)$ and figure $7(d)$.

The zip procedure is defined by the following operations:

1. Spreading: A partition of double length is created by setting $j:=2 j-1$ and leaving $C_{i}^{*}:=C_{i}($ for $i=1, \ldots, r$ and $j=1, \ldots, n)$.

2. Turning: The partition is turned upside down by setting $C_{i}^{* *}:=C_{r+1-i}^{*}$ (for $i=$ $1, \ldots, r)$.

3. Shifting: The partition is shifted left for the length of a certificate by setting $j:=j-n(\bmod 2 n)$ and $C_{i}^{* * *}:=C_{i}^{* *}($ for $i=1, \ldots, r$ and $j=1, \ldots, n)$.

4. Merging: Form a partition by merging the subsets resulting from the spreading and the shifting step by setting $C_{i}^{* * * *}:=C_{i}^{*} \cup C_{i}^{* * *}($ for $i=1, \ldots, r)$.

The definition of a zipped certificate is as follows:

Definition 4.1 A zipped certificate $Z W(r, k, 2 \times n)$ is a certificate obtained by applying the zip procedure on a certificate $W(r, k, n)$. 
(a)

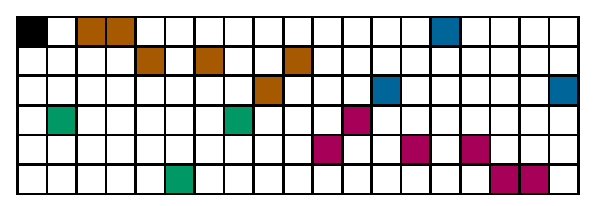

(b)

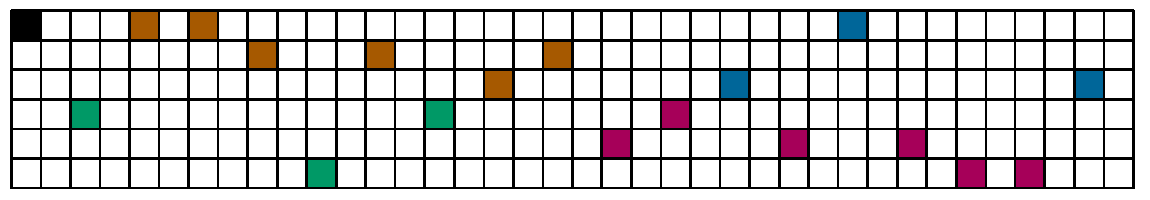

(c)

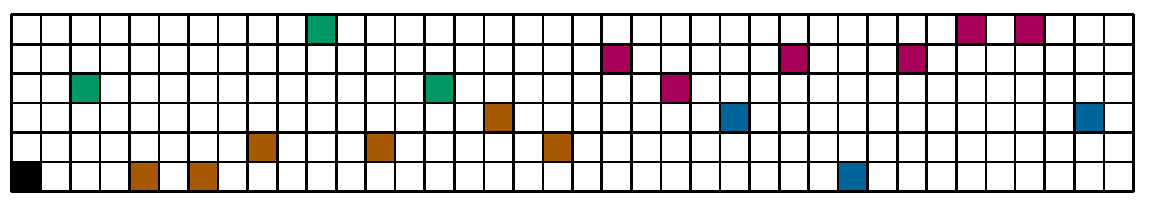

$(d)$

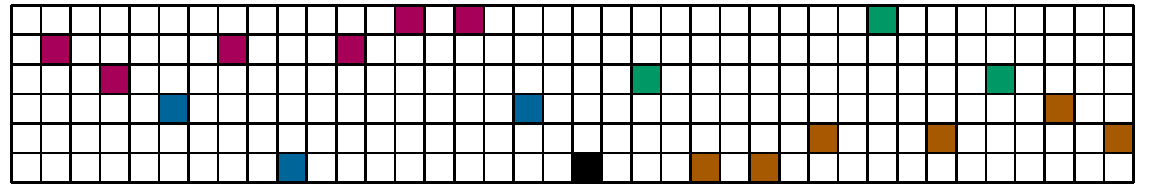

$(e)$

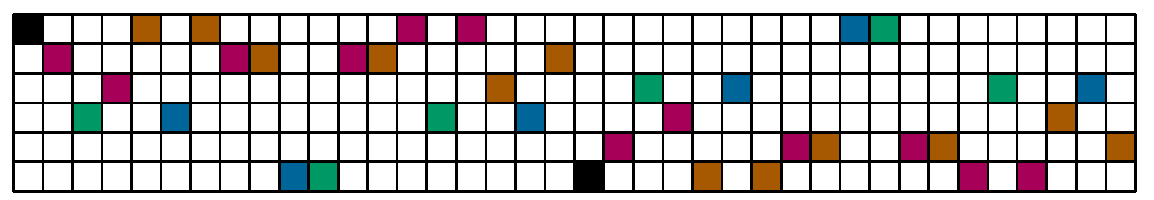

Figure 7: Illustrated example of zipping: $(a)$ a $c P W(6,3,19)$ certificate; $(b)$ result of spreading $(a)$ on all odd positions; $(c)$ result of turning $(b)$ upside down; $(d)$ result of shifting $(c)$ left with 19 positions; and $(e)$ results in a $Z c P W(6,3,2 \times 19)$ certificate by merging $(b)$ and $(d)$.

Some zipped certificates can be zipped again (using the original certificate length to shift) to obtain an even longer zipped certificate. Zipping more then twice did not result in useful certificates. An example of a second degree zipping of a certificate $c P W(2,5,11)$ is given in figure 8. The repetitive certificate of this result $\operatorname{ZZCPW}(2,5,4 \times 44+1)$ is the largest possible lower bound for $W(2,5)$.

\section{Results}

The observations from the previous section can be combined in a single procedure, the Cyclic Zipper method:

1. Cyclic certificate: Suppose a cyclic certificate of length $n$ is found - by power residue coloring (see section 4.2), or by any other technique.

2. Zip: Zip this solution $z$ times to obtain a new certificate of length $2 z \times n$.

3. Validate: Check if the zipped certificate is cyclic itself. 
4. Repeat: Create a repetitive cyclic certificate by appending the present certificate $(k-1)$ times and expand it with one additional number at the end.

(a)
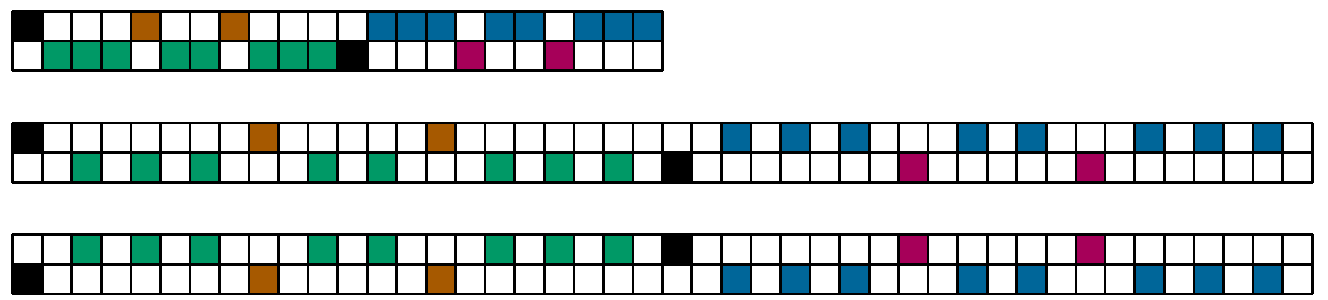

$(e)$

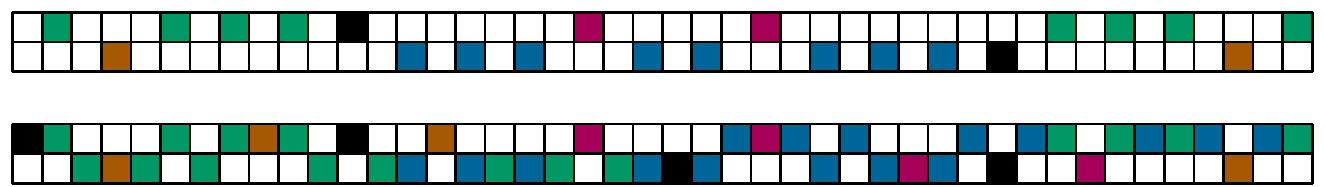

Figure 8: Illustrated example of second degree zipping: (a) a $Z c P W(2,5,2 \times 11)$ certificate obtained by power residue coloring followed by zipping; (b) result of spreading $(a)$ on all odd positions; $(c)$ result of turning $(b)$ upside down; $(d)$ result of shifting $(c)$ left with 11 positions; and $(e)$ results in a $Z Z c P W(2,5,4 \times 11)$ by merging $(b)$ and $(d)$.

With the Cyclic Zipper method we can determine the largest outcome of combining zipping with a specific cyclic certificate. First, certificates were constructed using power residue coloring. Then the cyclic certificates were zipped to find the largest certificates. The results are shown in table 3. The first column shows the Van der Waerden number. In the second and third column, the used prime for power residue coloring and the number of zippings are presented. The last column shows the lower bound obtained by the Cyclic Zipper method. The lower bounds in bold are improvements over the existing lower bounds.

Notice that new lower bounds were only found for partitions with an even number of subsets. Apparently, zipping is not effective for the odd values of $r$. Also note that with the Cyclic Zipper method all current lower bounds for the even values of $r$ can be obtained, except for $W(2,3)$.

Our website ${ }^{6}$ contains the raw certificates proving the improved lower bounds. Visualizations of the certificates that represent improved lower bounds for $W(4, k)$ and $W(6, k)$ are presented in Appendix A. Notice that the certificates that were zipped, contain an almost perfect double symmetry.

\footnotetext{
${ }^{6}$ http://www.st.ewi.tudelft.nl/sat/waerden.htm
} 
Table 3: Lower bounds reached with power residue coloring combined with zipping.

\begin{tabular}{|c|r|r|r|}
\hline$W(r, k)$ & used prime & zipped & result \\
\hline \hline$W(2,3)$ & - & - & - \\
\hline$W(2,4)$ & 11 & 0 & $>34$ \\
\hline$W(2,5)$ & 11 & 2 & $>177$ \\
\hline$W(2,6)$ & 113 & 1 & $>1131$ \\
\hline$W(2,7)$ & 617 & 0 & $>3703$ \\
\hline$W(2,8)$ & 821 & 1 & $>\mathbf{1 1 4 9 5}$ \\
\hline$W(2,9)$ & 2579 & 1 & $>\mathbf{4 1 2 6 5}$ \\
\hline$W(4,3)$ & 37 & 0 & $>75$ \\
\hline$W(4,4)$ & 349 & 0 & $>1048$ \\
\hline$W(4,5)$ & 2213 & 1 & $>\mathbf{1 7 7 0 5}$ \\
\hline$W(4,6)$ & 9133 & 1 & $>\mathbf{9 1 3 3 1}$ \\
\hline$W(4,7)$ & 32789 & 1 & $>\mathbf{3 9 3 4 6 9}$ \\
\hline$W(6,3)$ & 103 & 0 & $>207$ \\
\hline$W(6,4)$ & 3259 & 0 & $>9778$ \\
\hline$W(6,5)$ & 3967 & 2 & $>\mathbf{6 3 4 7 3}$ \\
\hline$W(6,6)$ & 31699 & 2 & $>\mathbf{6 3 3 9 8 1}$ \\
\hline
\end{tabular}

\section{Conclusions}

In this paper we presented a method to construct lower bounds for Van der Waerden numbers. The final results are shown in table 4 .

Table 4: Known Van der Waerden numbers and known and improved lower bounds.

\begin{tabular}{|c|r|r|r|r|r|r|r|}
\hline$r \backslash k$ & $\mathbf{3}$ & $\mathbf{4}$ & $\mathbf{5}$ & $\mathbf{6}$ & $\mathbf{7}$ & $\mathbf{8}$ & $\mathbf{9}$ \\
\hline \hline $\mathbf{2}$ & 9 & 35 & 178 & $>1131$ & $>3703$ & $>\mathbf{1 1 4 9 5}$ & $>\mathbf{4 1 2 6 5}$ \\
\hline $\mathbf{3}$ & 27 & $>292$ & $>1209$ & $>8886$ & $>43855$ & $>238400$ & \\
\hline $\mathbf{4}$ & 76 & $>1048$ & $>\mathbf{1 7 7 0 5}$ & $>\mathbf{9 1 3 3 1}$ & $>\mathbf{3 9 3 4 6 9}$ & & \\
\hline $\mathbf{5}$ & $>125$ & $>2254$ & $>24045$ & $>246956$ & & & \\
\hline $\mathbf{6}$ & $>207$ & $>9778$ & $>\mathbf{6 3 4 7 3}$ & $>\mathbf{6 3 3 9 8 1}$ & & & \\
\hline
\end{tabular}

This paper presented a way to expand the size of a certificate by zipping. Combining the existing knowledge to create cyclic certificates with this zipping technique resulted in the Cyclic Zipper method. Using this procedure we determined seven improved lower bounds for the Van der Waerden numbers.

The technique of power residue coloring to create cyclic certificates that could be zipped does not only result in certificates for the improved lower bounds, but also provides certificates for all the other proved and currently known lower bounds for Van der Waerden numbers with even $r$. The only exception is the lower bound of the Van der Waerden number $W(2,3)$. 
These results ignite some discussion. As Kouril et al. [11] notice, $W(2, k)$ can - for small $k$ - be roughly estimated by $k \times W(2, k-1)$. If this also holds for larger $k$, one would expect that the lower bounds for $W(2,7), W(2,8)$ and $W(2,9)$ could be improved significantly. Besides, zipping cyclic certificates was not effective to partition $\{1, \ldots, n\}$ into an odd number of subsets. Perhaps the regularity posed by Kouril et al. is a coincidence. But, especially the fact that the procedure was not effective for odd values of $r$ suggests our method needs one more generalization step.

\section{Acknowledgments}

We would like to thank Anne-Aimee Bun and Michel Meulpolder for cooperating in the research project. We would also like to thank Michal Kouril and John Franco for introducing us to the writings of Rabung [13].

\section{References}

[1] Michael D. Beeler and Patrick E. O’Neil, Some new Van der Waerden numbers. In Discrete Mathematics Vol. 28, pages 135-146, 1979

[2] E. Berlekamp, A construction for partitions which avoid long arithmetic progressions. In Canadian Mathematical Bulletin Vol. 11, pages 409-414, 1968

[3] V. Chvátal, Some unknown Van der Waerden numbers. In R.K. Guy et al., eds., Combinatorial Structures and their Applications, pages 31-33, (Gordon and Breach, New York, 1970)

[4] M. R. Dransfield, L. Liu, V. W. Marek and M. Truszczynski, Satisfiability and Computing van der Waerden Numbers. In The Electronic Journal of Combinatorics. Vol. 11 (1), 2004

[5] P. Erdős and R. Rado, Combinatorial theorems on classifications of subsets of a given set. In Proceedings of London Mathematical Society Vol. 2, pages 417-439, 1952

[6] H. Fredricksen and M.M. Sweet, Symmetric sum-free partitions and lower bounds for Schur numbers. Electronic Journal of Combinatorics 7 (1) R32, pages 1-9,2000

[7] C.P. Gomes and M. Sellmann, Streamlined constraint reasoning. CP04, Lecture Notes in Computer Science 3258: 274-289, 2004.

[8] T. Gowers, A new proof of Szemeredi theorem. In Geometric and Functional Analysis. Vol. 11, pages 465-588, 2001

[9] R. N. Greenwell and B. M. Landman, On the existence of a reasonable upper bound for the van der Waerden numbers. In J. Combin. Theory Ser. A. Vol. 50, pages 82-86, 1989

[10] M.J.H. Heule, J.E. van Zwieten, M. Dufour, and H. van Maaren. March_eq: Implementing Additional Reasoning into an Efficient Lookahead Sat Solver. SAT 2004 Springer LNCS 3542, pages 345-359, 2005 
[11] M. Kouril and J. Franco, Resolution Tunnels for Improved SAT Solver Performance. In Proc. of 8th International Conference on Theory and Applications of Satisfiability Testing. pages 143-157, 2005

[12] B.M. Landman and A. Robertson, Ramsey Theory on the Integers (Student Mathematical Library, V. 24) American Mathematical Society, 2004

[13] John, R. Rabung, Some Progression-free Partitions Constructed Using Folkman's Method. In Canadian Mathematical Bulletin Vol. 22 (1), pages 87-91, 1979

[14] S. Shelah, Primitive recursive bounds for van der Waerden numbers. In J. Amer. Math. Soc. Vol. 1, pages 683-697, 1988

[15] H. Y. Song, H. Taylor and S. W. Golomb, Progressions in Every Two-coloration of Z n. In J. Combin. Theory Ser. A. Vol. 61 (2), pages 211-221, 1992

[16] R.S. Stevens and R. Shantaram, Computer generated Van der Waerden partitions. In Mathematics of Computation Vol. 32 (142), pages 635-636, 1978

[17] E. Szemerédi, On sets of integers containing no k elements in arithmetic progression. In Acta Arithmetica Vol. 27, pages 199-243, 1988

[18] B.L. van der Waerden, Beweis einer Baudet'schen Vermutung. In Nieuw Archief voor Wiskunde Vol. 15, pages 212-216, 1927

\section{A Appendix}

Here the visualizations of the certificates, improving the lower bounds for $W(4, k)$ and $W(6, k)$, are shown. The cycles of a zipped cyclic certificate are exact copies of each other which exactly overlap. For the sake of clarity, only the first cycle is visualized.

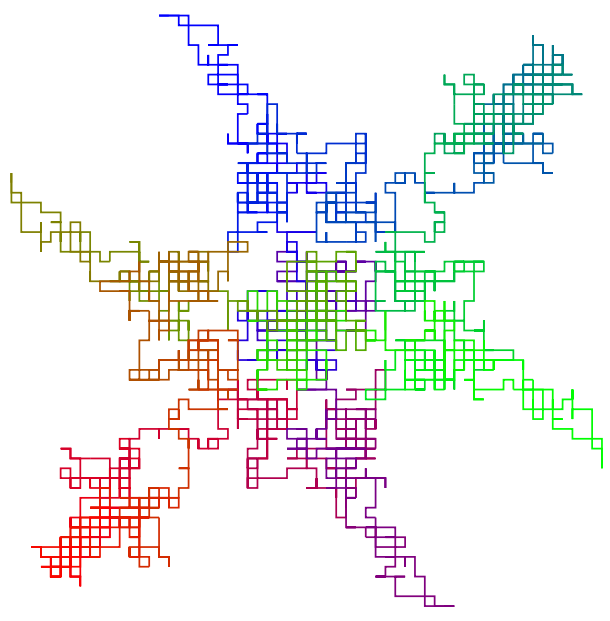

Figure 9: Graphical representation of a cycle of a $Z C W(4,5,17705)$ 


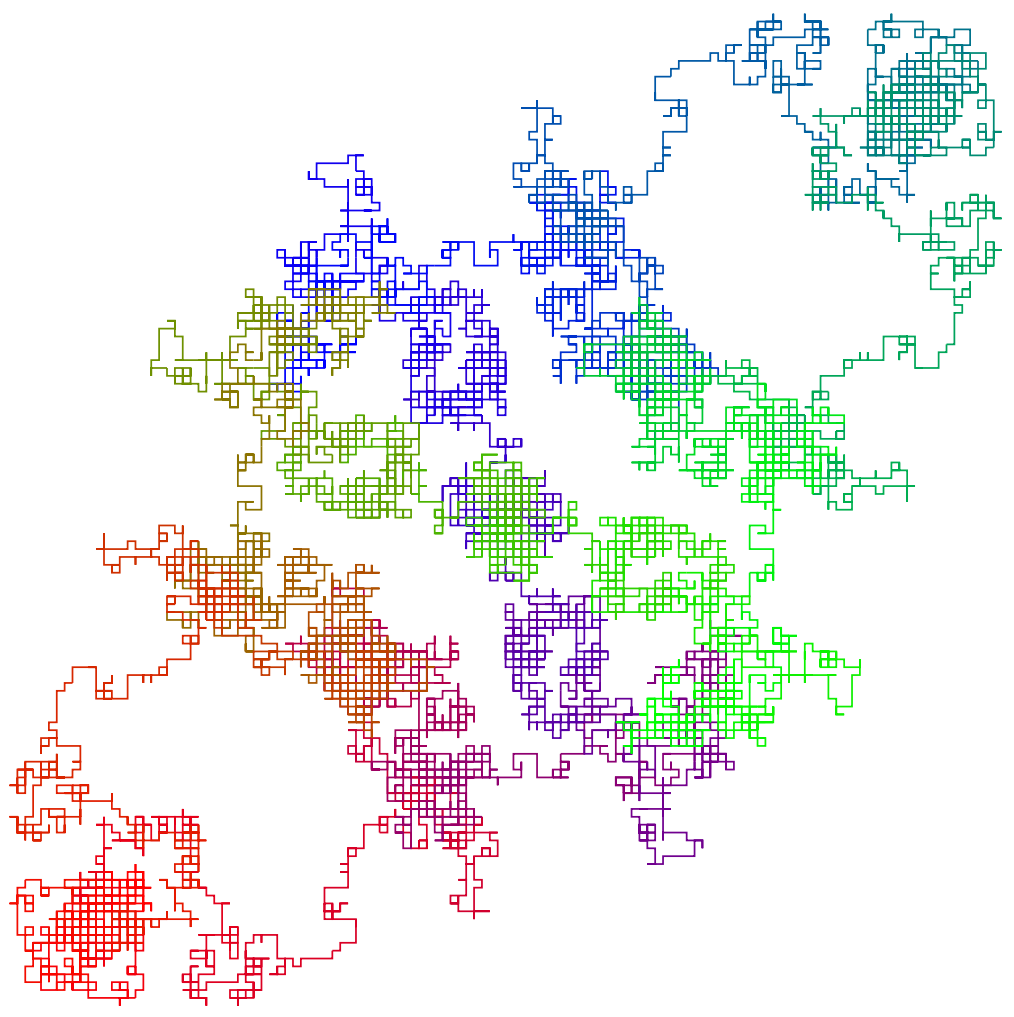

Figure 10: Graphical representation of a cycle of a $Z C W(4,6,91331)$ 


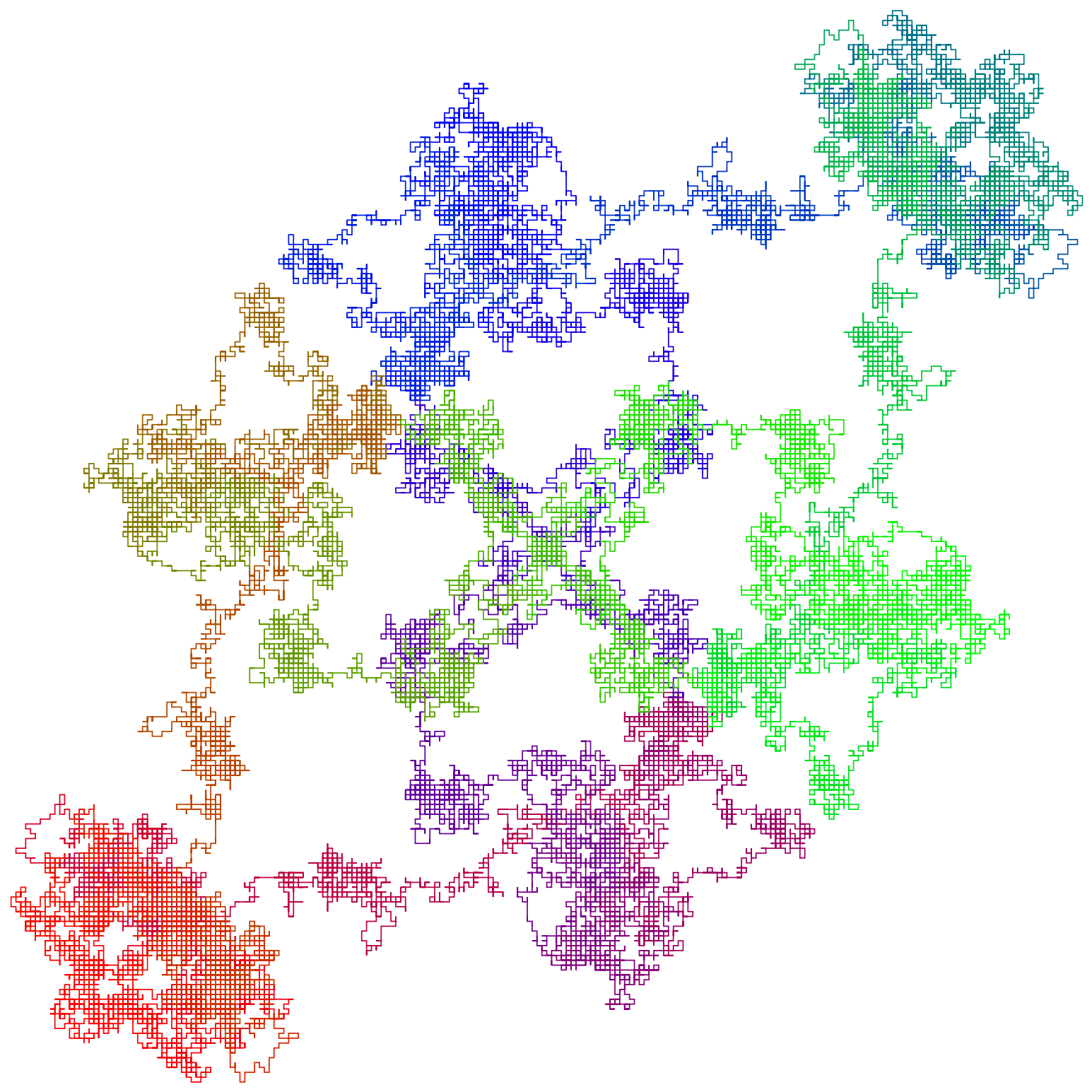

Figure 11: Graphical representation of a cycle of a $Z C W(4,7,393469)$ 


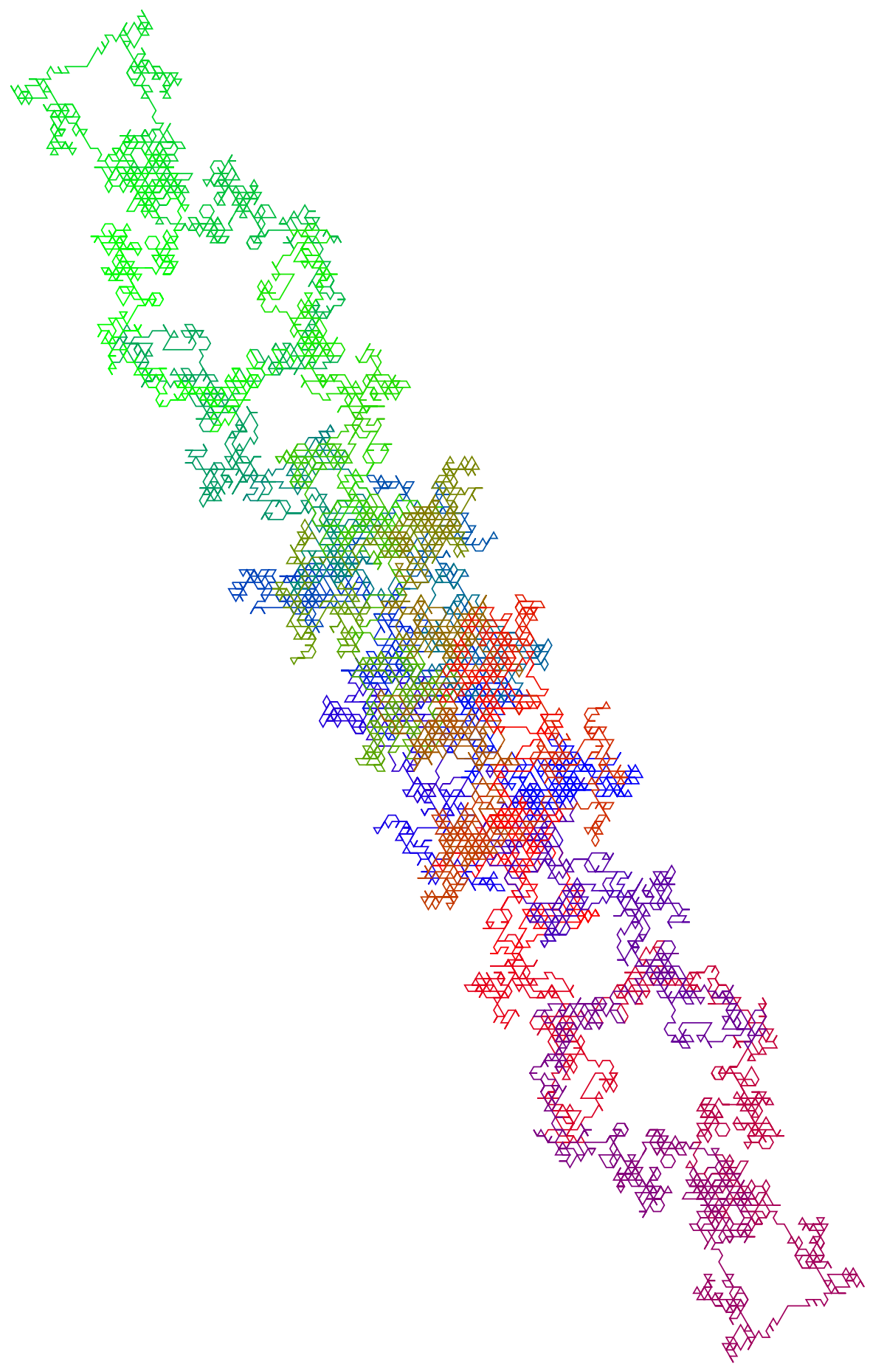

Figure 12: Graphical representation of a cycle of a $Z Z C W(6,5,63473)$ 


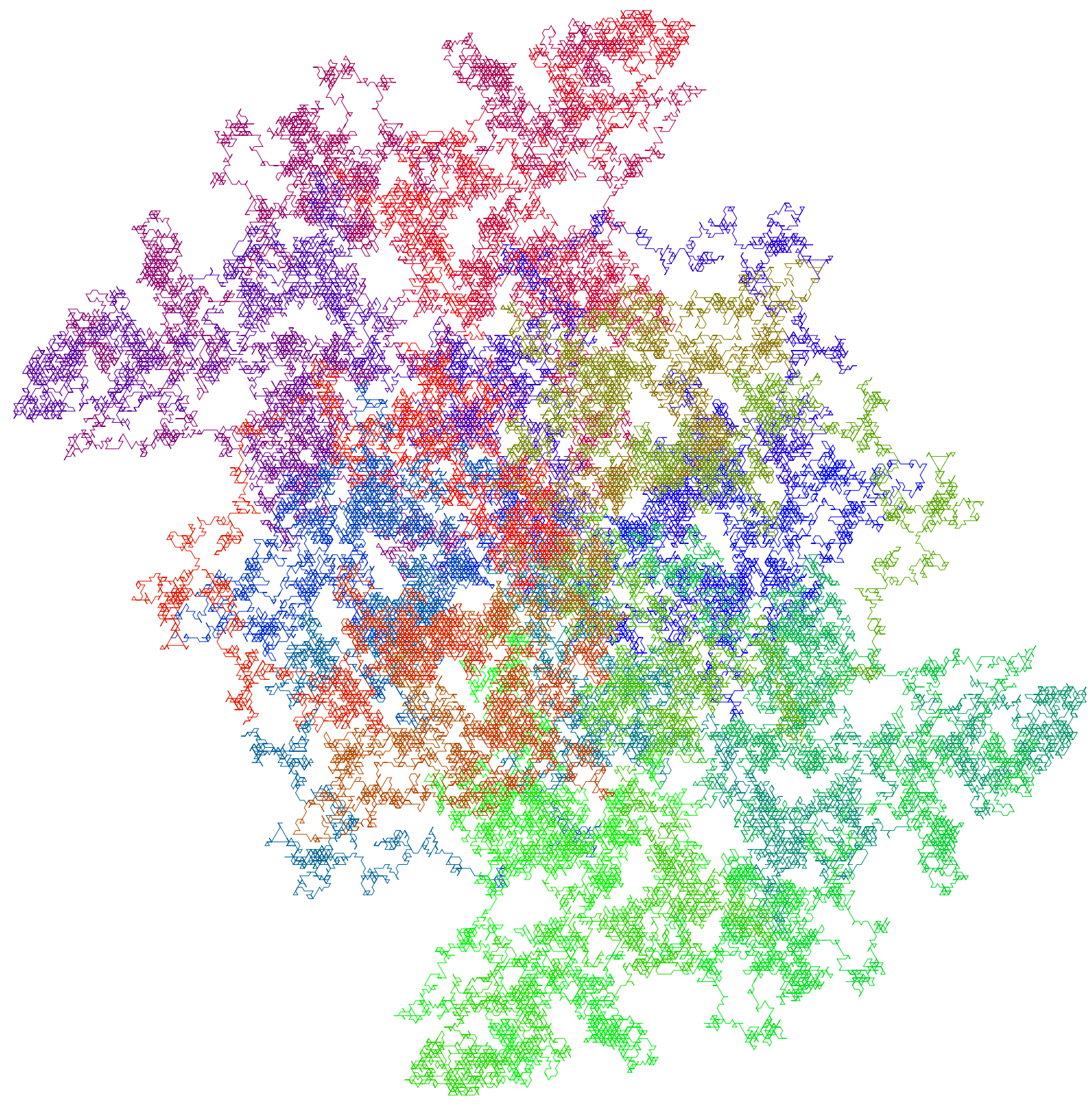

Figure 13: Graphical representation of a cycle of a $Z Z C W(6,6,633981)$ 